\title{
Logaritma Fonksiyonunun İrrasyonel Fonksiyon İle Temsili ve Bu Temsile Dayalı Üstel Fonksiyon Elde Edilmesi Üzerine
}

\author{
Müslüm Özış1k ${ }^{1 *}$ \\ 1*Yıldız Teknik Üniversitesi, Kimya-Metalürji Fakültesi, Matematik Mühendisliği Bölümü, İstanbul, Türkiye, (ORCID: 0000-0001-6143-5380), ozisik@yildiz.edu.tr
} (İlk Geliş Tarihi 30 Nisan 2021 ve Kabul Tarihi 24 Temmuz 2021)

(DOI: $10.31590 /$ ejosat.930694)

ATIF/REFERENCE: Özışı, M., (2021). Logaritma Fonksiyonunun İrrasyonel Fonksiyon İle Temsili ve Bu Temsile Dayalı Üstel Fonksiyon Oluşturulması. Avrupa Bilim ve Teknoloji Dergisi, (25), 542-549.

Öz

$\mathrm{Bu}$ çalışmada matematikte çok yaygın bir kullanım alanına sahip olan en temel fonksiyonlardan olan logaritma fonksiyonunun irrasyonel bir fonksiyon ile temsil edilmesi üzerine çalışılmıştır. Bu amaçla logaritma fonksiyonu yerine kullanılabilecek irrasyonel bir fonksiyon önerisi yapılmış, uygun matematik ve nümerik analiz tanım ve yöntemleri çerçevesinde önerilen fonksiyon elde edilmiştir. Elde edilen irrasyonel fonksiyon üzerinden ters fonksiyonu oluşturulmak suretiyle üstel fonksiyonlar için yeni bir yaklaşım elde edilmiştir. Sayısal sonuçlar ve grafikler elde edilerek gerekli değerlendirmeler yapılmıştır.

Anahtar Kelimeler: Logaritma, Doğal Logaritma, Napier, Euler.

\section{On the Representation of the Logarithm Function by the Irrational Function and the Obtaining of an Exponential Function Based on This Representation}

\begin{abstract}
In this study, the representation of the logarithm function, which is one of the most basic functions that has a very common usage area in mathematics, has been studied with an irrational function. For this purpose, an irrational function that can be used instead of a logarithm function has been proposed, and the proposed function has been obtained within the framework of appropriate mathematical and numerical analysis definitions and methods. A new approach has been obtained for exponential functions by constructing the inverse function on the obtained irrational function. Necessary evaluations were made by obtaining numerical results and graphics.
\end{abstract}

Keywords: Logarithm, Natural Logarithm, Napier, Euler.

* Sorumlu Yazar: Yıldız Teknik Üniversitesi, Kimya-Metalürji Fakültesi, Matematik Mühendisliği Bölümü, İstanbul, Türkiye_ozisik@yildiz.edu.tr 


\section{Giriş}

Logaritma fonksiyonu matematik ve bilimin en temel ve vazgeçilmez fonksiyonlarından biridir. Logaritma hesabının temelini teşkil eden üslü sayıların logaritmasını ilk tanımlayan Michael Stifel (1487-1567) olmuş aynı zamanda 2 sayısının kuvvetlerini incelemiştir. Ancak farklı birçok taban kullanmak suretiyle logaritmayı gerçek anlamda kullanan John Napier (1550-1617) olmuştur. Napier'in logaritma ile olan çalışmaları genel olarak cebire dayanmaktaydı ve logaritma ile ilgili ilç çalışmasını 1614 yılında "A Description of the Wonderful Table of Logarithms (Harika Logaritma Tablosunun bir Açıklaması)" adı altında yayınladı. Napier bu çalışmasında Yunanca "logos" ve "arithmo" kelimelerini sayılar ve logaritmalarını ifade etmek üzere bir arada "logaritma" olarak kullanmış, logaritmayı da "doğal” ve "yapay" olarak isimlendirmiştir. (Günümüzde $\mathrm{y}=\operatorname{Ln}_{\mathrm{e}} \mathrm{x}$ doğal logaritma ifadesi aynı zamanda Napier logaritması olarak da bilinmektedir.) Aynı dönemde Henry Briggs (1551-1630) ve Joost Burgi (1552-1632) de üstel işlemlerle uğraşmış olan matematikçiler arasındadır. Ancak Burgi'nin logaritma ile ilgili çalışmaları Napier'den farklı olarak cebire değil geometriye dayanmaktayd. Günümüzde kullanılan logaritma ise bilime Leonhard Euler (1707-1783) tarafindan kazandırılmıştır. Napier logaritmasında da kullanılan "e sabitinin" ilk defa 1727 yılında ifade edilmesi, 1737 yılında e sayısının irrasyonel olduğunun gösterilmesi, 1748 yılında da virgülden sonraki ilk 18 basamağının hesaplanması Euler tarafindan logaritma hesabına ilişkin yapılmış çalışmalardan bazıları olup (Boyer 1991; Havil, 2003; Kathleen ve Montelle, 2011; Bruce, 2000; Fauvel, 2000; Rice ve ark., 2017) kaynaklarında yer almaktadirlar.

Gerek doğal logaritma gerekse logaritma fonksiyonu matematik ve mühendislik alanında temsil edilen birçok problemin içerisindeki yaygın kullanımına bağlı olarak her daim geniş bir ilgi alanına sahip olmuştur. Doğal logaritmanın Gaussian Hipergeometrik Fonksiyonlarla temsil edilmesi (Nofal, 2006), logaritmanın interpolasyon ile hesaplanması (Gautschi, 2008), qlogaritması için irrasyonel ölçümleme teknikleri üzerinde yapılan çalışmalar (Matala-Aho ve ark., 2005; Koelink ve Assche, 2009), logaritmik diferansiyel formlar, rezüdüler ve vektör alanları ile ilgili (Aleksandrov, 2014; Pol, 2018; Tajima ve Nabeshima, 2021) çalışmaları örnek olarak gösterilebilir. Doğal olarak diğer mühendislik alanlarından farklı olarak matematiğin temelini oluşturan bazı klasik kavram ve konularda çoğu zaman kısa zaman dilimleri içerisinde köklü bir tanım, anlam değişikliği olmaz. Daha ziyade uygulama alanları ve tekniklere ilişkin genişlemeler, yorumlar ve yöntemlere dair farklılıklar söz konusu olur. Bu yönüyle logaritma ve üstel işlemler bu alanlardan biridir.

$\mathrm{Bu}$ çalışmada, yukarıda kısaca özetlenen, matematik, mühendislik ve bilimin en temel kavram ve fonksiyonlarından olan logaritma ve üstel fonksiyona dair çalışma yapılmıştır. Logaritma fonksiyonunun irrasyonel fonksiyon ile temsiline ilişkin olarak fonksiyon önerisinde bulunulmuş, önerilen fonksiyon tanımlanmış, daha sonra bu fonksiyon uygun matematiksel ve nümerik yöntemler ile elde edilerek logaritma fonksiyonunun irrasyonel fonksiyon ile temsil edilebileceği sayısal ve grafiksel sonuçlarla gösterilmiştir. Sonrasında elde edilen fonksiyona bağlı olarak ters fonksiyonu elde edilmek suretiyle üstel işlemler için de kullanılabilecek üstel fonksiyon ifade edilmiş ve grafiksel olarak matematikte mevcut durumda kullanılan üstel fonksiyon ile karşılaştarılmıştır.

\section{Fonksiyonun Tanımlanması ve Çözüm}

\subsection{Logaritma fonksiyonunun tanımı}

Matematikte doğal logaritma fonksiyonu genel olarak;

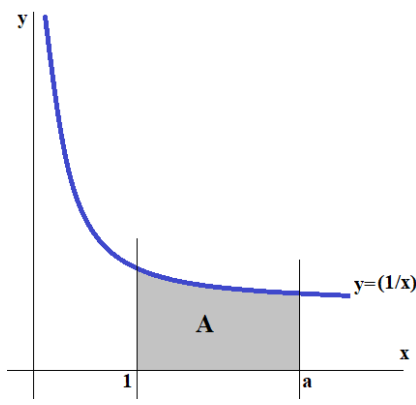

Şekil 1. Logaritmanın bir eğrinin altındaki alan olarak ifadesi

şekildeki gibi $\mathrm{y}=(1 / \mathrm{x})$ eğrinin altında ve $(\mathrm{y} \geq 0,1 \leq \mathrm{x} \leq \mathrm{a})$ ile sınırlı olan düzlemsel bölgenin alanı olarak

$A=\int_{1}^{a}\left(\frac{1}{x}\right) d x$

$A=\left.\operatorname{Ln} x\right|_{x=1} ^{a}$

$\mathrm{A}=\mathrm{Lna}$

şeklinde ifade edilir. Dolayısıyla doğal logaritma fonksiyonunun genel ifadesi

$0 \leq \mathrm{x}<\infty ; \mathrm{e} \cong 2,718281 \ldots$ olmak üzere,

$y=f(x)=\operatorname{Ln}_{e} x=\operatorname{Ln} x$

olarak verilir.

Doğal logaritma fonksiyonuna benzer olarak logaritma fonksiyonu ise genel olarak bütün matematik kitaplarında;

$\forall \mathrm{a}, \mathrm{x}>0$ ve $\mathrm{a} \neq 1$ (a : logaritma tabanı) olmak üzere;

$y=f(x)=\log _{a} x$

olarak tanımlanmış olup grafikleri aşağıdaki gibidir. (Kreyszig, 1993; Andrews ve ark., 1999; Thomas ve ark. 2010).

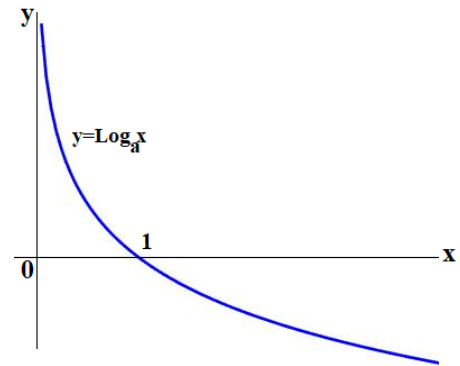

Şekil 2. $\mathrm{x}>0,0<\mathrm{a}<1$ için $\log _{a} x$ grafiği

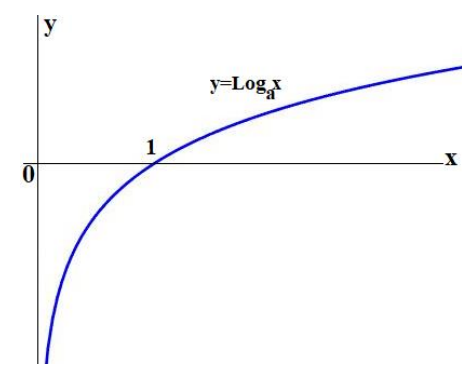

Şekil 3. $x>0, a>1$ için $\log _{\mathrm{a}} \mathrm{x}$ grafiğ $\mathrm{i}$

\subsubsection{Irrasyonel fonksiyon önerilmesi ve elde edilmesi}

Logaritma fonksiyonunun Şekil 2. ve Şekil 3. ile verilen genel grafikleri dikkate alındığında bu eğrilerin benzer irrasyonel 
eğrilerle temsil edilebileceği (genel anlamda $\sqrt[q]{\mathrm{x}}-1, \mathrm{x}>0$, $\mathrm{q} \in \mathrm{Z}^{+}$formunda) fikri hareket noktasının temelini oluşturmuştur.

Dolayısıyla;

SLog ${ }_{a} x$ fonksiyonu, $y=f(x)=\log _{a} x$ fonksiyonun temsili için önerilen irrasyonel fonksiyon olmak üzere bu fonksiyon,

$y=\operatorname{SLog}_{a} x=[\sqrt[p]{x}-1] \cdot K_{a}=f_{a}(x)$

olarak verilmiş olsun. Burada;

$\mathrm{a}>0, \quad \mathrm{a} \neq 1$ olmak üzere logaritma tabanını, $\mathrm{x}>0$ olarak logaritması alınacak olan sayıyı, $\mathrm{p}=2^{\mathrm{n}}, \mathrm{n} \in \mathrm{Z}^{+}$olmak üzere kök derecesini, $\mathrm{K}_{\mathrm{a}}$ ise logaritma tabanına (a'ya) bağlı bir reel sayıyı ifade etmektedir. (4) ile önerilen irrasyonel fonksiyondaki $[\sqrt[p]{\mathrm{x}}-1]$ ifadesi logaritma fonksiyonu için $\forall \mathrm{a}>0, \mathrm{a} \neq 1$ için $\left.\operatorname{SLog}_{\mathrm{a}} \mathrm{x}\right|_{\mathrm{x}=1}=\left.\mathrm{f}_{\mathrm{a}}(\mathrm{x})\right|_{\mathrm{x}=1}=0$ koşulunu sağlaması içindir. (4) ile önerilmiş olan irrasyonel fonksiyon logaritma fonksiyonunun temsili için önerilmiş olduğundan;

$y=\log _{a} x \cong[\sqrt[p]{x}-1] \cdot K_{a}=f_{a}(x)$

olarak yazabiliriz.

$\log _{\mathrm{a}} \mathrm{x}$ fonksiyonunun tanımı gereği $\forall \mathrm{a}, \mathrm{x}>0, \mathrm{a} \neq 1$, ve $\mathrm{c}>0$, $\mathrm{c} \neq 1$ olmak üzere (5) ifadesinden;

$\log _{a} \mathrm{x} \cong[\sqrt[p]{\mathrm{x}}-1] \cdot \mathrm{K}_{\mathrm{a}}=\mathrm{f}_{\mathrm{a}}(\mathrm{x})$

$\log _{\mathrm{c}} \mathrm{x} \cong[\sqrt[\mathrm{p}]{\mathrm{x}}-1] \cdot \mathrm{K}_{\mathrm{c}}=\mathrm{f}_{\mathrm{c}}(\mathrm{x})$

$\log _{c} a \cong[\sqrt[p]{a}-1] \cdot K_{c}=f_{c}(a)$

olarak yazılsın.

$\log _{\mathrm{a}} \mathrm{x}$ logaritma fonksiyonu için bilinen taban değiştirme özelliği ve (6) eşitlikleri de kullanılmak suretiyle;

$$
\begin{aligned}
\log _{a} x & =\frac{\log _{c} x}{\log _{c} a} \\
& =\frac{f_{c}(x)}{f_{c}(a)}
\end{aligned}
$$

olarak yazılabilir. (6) ifade ile;

$f_{c}(x)=[\sqrt[p]{x}-1] \cdot K_{c}$

ve

$$
f_{c}(a)=[\sqrt[p]{a}-1] \cdot K_{c}
$$

olarak verildiğinden;

$\log _{a} x=\frac{f_{c}(x)}{f_{c}(a)}=\frac{[\sqrt[p]{x}-1] \cdot K_{c}}{[\sqrt[p]{a}-1] \cdot K_{c}}$ $\log _{a} x=\frac{[\sqrt[p]{x}-1] \cdot K_{c}}{[\sqrt[p]{a}-1] \cdot K_{c}}$

$\log _{a} x=\frac{[\sqrt[p]{x}-1]}{[\sqrt[p]{a}-1]}$

$\log _{a} x=\frac{\sqrt[p]{x}-1}{\sqrt[p]{a}-1}$

olarak bulunur. (5) ve (7) ifadeleri birlikte dikkate alınırsa $\mathrm{K}_{\mathrm{a}}$ reel sayıs1;

$[\sqrt[p]{x}-1] \cdot K_{a}=\frac{\sqrt[p]{x}-1}{\sqrt[p]{a}-1}$

$\mathrm{K}_{\mathrm{a}}=\frac{1}{\sqrt[p]{\mathrm{a}}-1}$

olarak bulunur. $\left(\mathrm{K}_{\mathrm{a}} \in \mathrm{R} ;\left(\mathrm{a}>0, \mathrm{a} \neq 1, \mathrm{p}=2^{\mathrm{n}}, \mathrm{n} \in \mathrm{Z}^{+}\right)\right.$

Dolayısıyla (4) ile önerilmiş olan irrasyonel fonksiyon;

$\operatorname{SLog}_{a} x=\frac{\sqrt[p]{x}-1}{\sqrt[p]{a}-1}$

olarak yazılabilecektir. $\left(\forall \mathrm{a}, \mathrm{x}>0, \mathrm{a} \neq 1, \mathrm{p}=2^{\mathrm{n}}, \mathrm{n} \in \mathrm{Z}^{+}\right)$

$\mathrm{Bu}$ durumda geriye (10) ifadesindeki $\mathrm{p}=2^{\mathrm{n}}, \mathrm{n} \in \mathrm{Z}^{+}$kök derecesinin belirlenmesi kalmaktadır. (4) ifadesiyle önerilen irrasyonel fonksiyon logaritma fonksiyonu yerine önerilmiş olduğundan $\forall \mathrm{a}, \mathrm{x}>0, \mathrm{a} \neq 1$ olmak üzere,

$\log _{\mathrm{a}} \mathrm{x} \cong \operatorname{SLog}_{\mathrm{a}} \mathrm{x}$

ifadesinin gerçekleşmesi gerekir. Bir başka deyişle

$$
\begin{aligned}
& \operatorname{Lim}_{k \rightarrow n}\left|\log _{a} x-S \log _{a} x\right| \\
& \operatorname{Lim}_{k \rightarrow n}\left|\log _{a} x-\frac{\sqrt[k]{x}-1}{\sqrt[k]{a}-1}\right|
\end{aligned}
$$

ifadesinin limit değerinin sıfıra yaklaşması yani;

$$
\operatorname{Lim}_{k \rightarrow n}\left|\log _{a} x-\frac{\sqrt[k]{x}-1}{\sqrt[k]{a}-1}\right| \rightarrow 0
$$

olması gerekir.

Söz konusu limit işlemi için uygun algoritma ve nümerik yöntemler uygulandığında değişik $\mathrm{n} \in \mathrm{Z}^{+}$değerleri için (13) ifadesinin limit değerinin sıfır olduğu görülecektir. (4) ile önerilmiş olan $\quad \operatorname{LLg}_{\mathrm{a}} \mathrm{x}$ irrasyonel fonksiyonun $\log _{\mathrm{a}} \mathrm{x}$ fonksiyonu için temsili noktasında bir çalışma hedeflendiğinden $\left|\log _{\mathrm{a}} \mathrm{x}-\operatorname{SLog}_{\mathrm{a}} \mathrm{x}\right|$ farkının olabildiğince en büyük hassasiyette sıfıra eşit olması elde edilen sonucun geçerliliği noktasında önem taşımaktadır. Bu nedenle $\mathrm{n}$ için elde edilen sayısal sonuçların elde 
edilmesi aşamasında $\left|\log _{\mathrm{a}} \mathrm{x}-\mathrm{S} \log _{\mathrm{a}} \mathrm{x}\right|<10^{-7}$ olacak şekilde algoritma kurulmuş olup (13) ifadesindeki kök derecesine ilişkin olarak,

$$
\mathrm{n}=30 \quad ; \quad \mathrm{p}=2^{30}
$$

olarak bulunmuştur.

Böylece $\log _{a} x$ logaritma fonksiyonunun yerine (4) ile önerilen irrasyonel $\operatorname{SLog}_{\mathrm{a}} \mathrm{x}$ fonksiyonu

$\operatorname{SLog}_{a} x=\frac{\sqrt[2]{x} \sqrt{x}-1}{\sqrt[30]{a}-1}$

olarak yazılmış olmaktadır. $(\forall \mathrm{a}, \mathrm{x}>0, \mathrm{a} \neq 1)$

$\log _{a} x$ logaritma fonksiyonun irrasyonel temsili için önerilen ve (15) ile elde edilen $\operatorname{SLog}_{\mathrm{a}} \mathrm{x}$ irrasyonel fonksiyonununn grafiği ile $\log _{\mathrm{a}} \mathrm{x} \operatorname{logaritma}$ fonksiyonun grafiği Şekil 4. ve Şekil 5. ile birlikte verilmiştir.
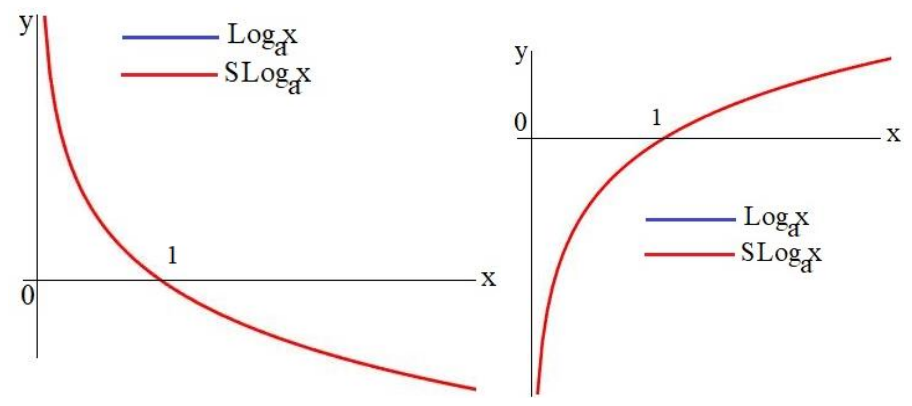

Şekil 4. $\mathrm{x}>0,0<\mathrm{a}<1$ Şekil 5. $x>0, a>1$ için için $\log _{a} x$ ve SLog $x$ grafikleri

$\log _{a} x$ ve SLog $x$ grafikleri

Şekil 4. ve Şekil 5. ile verilen grafiklerden görüleceği üzere irrasyonel $\operatorname{SLog}_{\mathrm{a}} \mathrm{x}$ fonksiyonu ile $\log _{\mathrm{a}} \mathrm{x}$ fonksiyonu örtüşmekte, $x>0$ için türevli ve sürekli olduğu görülmektedir.

\subsection{2. Üstel fonksiyonun elde edilmesi}

Matematikte üstel fonksiyon genel olarak logaritma fonksiyonun ters fonksiyonu olarak ifade edilmekte ve $\log _{\mathrm{a}} \mathrm{x}$ fonksiyonu için üstel fonksiyon ise;

$\mathrm{a} \in \mathrm{R}^{+}-\{1\}$ ve $\mathrm{a} \in \mathrm{R}^{+}$

olmak üzere;

$y=a^{x}$

şeklinde tanımlanmaktadır. $\mathrm{y}=\mathrm{a}^{\mathrm{x}}$ üstel fonksiyon ve $\mathrm{y}=\log _{\mathrm{a}} \mathrm{x} \operatorname{logaritma}$ fonksiyonlarının grafikleri ise;

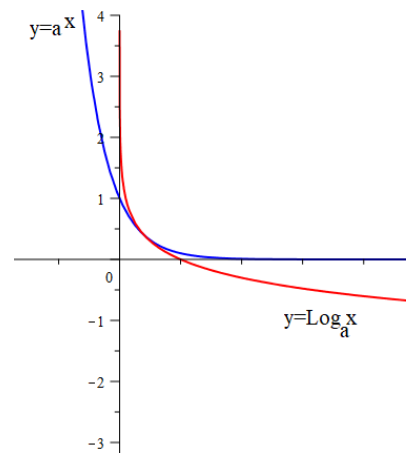

Şekil 6. $0<\mathrm{a}<1$ için logaritma ve üstel fonksiyon grafiği.

şeklindedir. Dolayısıyla (15) ile elde edilmiş olan $\operatorname{SLog}_{\mathrm{a}} \mathrm{x}$ irrasyonel fonksiyonu için de matematikte ters fonksiyonların elde edilmesine dair bilinen işlemlerin yapılması suretiyle;

$y=f(x)$ için $x=f^{-1}(y)$ ters fonksiyonu olmak üzere;

$\operatorname{SLog}_{a} x=\frac{2^{30} \sqrt{x}-1}{\sqrt[3]{a}-1}$

irrasyonel fonksiyonu için ters fonksiyonu (üstel fonksiyonu)

$\operatorname{eSLog}_{\mathrm{a}} \mathrm{x}=\left[\mathrm{x} \cdot\left(\sqrt[3^{30}]{\mathrm{a}}-1\right)+1\right]^{2^{30}} ;(\mathrm{x}>0, \mathrm{a}>0)$

olarak yazilabilir. ( $\operatorname{eSLog} \operatorname{ag}_{\mathrm{a}} \mathrm{x}$ notasyonu $\operatorname{SLog}_{\mathrm{a}} \mathrm{x}$ fonksiyonunun ters fonksiyonu olarak kullanılmıştır.)

(16) ifadesi de $\mathrm{y}=\mathrm{a}^{\mathrm{x}}$ üstel fonksiyonunu temsilen elde edilmiş olan üstel fonksiyon olduğundan (16) ifadesindeki a ve x sırasıyla üs alma işlemine ait taban ve üssü ifade etmektedir.

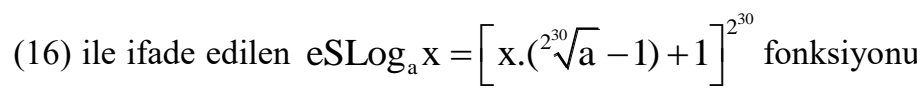
için de grafikleri çizdirilecek olursa;

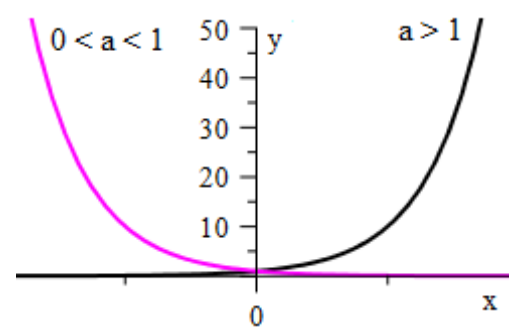

Şekil 8. eSLog $\mathrm{a}$ üstel fonksiyonu grafiği.

Şekil 6.-Şekil $7\left(\mathrm{y}=\mathrm{a}^{\mathrm{x}}\right)$ ve Şekil 8. $\left(\operatorname{eSLog}_{\mathrm{a}} \mathrm{x}\right)$ üstel fonksiyon grafikleri birlikte çizdirilecek olursa;

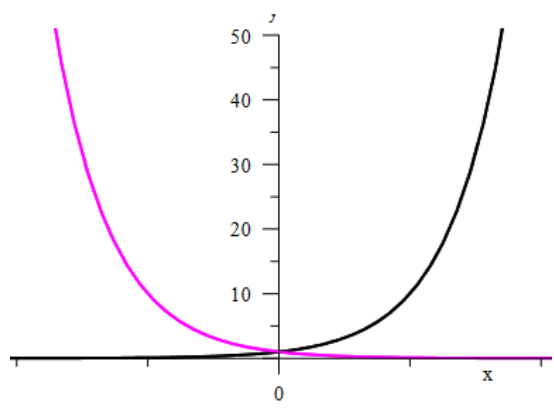

Şekil 9.- Şekil 6.-Şekil 7. ve Şekil 8. grafikleri 


\section{Araştırma Sonuçları ve Tartışma}

\subsection{Sayısal sonuçların elde edilmesi}

- Şekil 4. ve Şekil 5. ile logaritma fonksiyonu $\left(\log _{a} x\right)$ ile önerilen irrasyonel fonksiyona $\left(\operatorname{SLog}_{\mathrm{a}} \mathrm{x}\right)$ ait grafikler birlikte verilmek suretiyle grafiklerin örtüşmüş olduğu, Tablo 1.-Tablo 4. ile her iki fonksiyon için de değişik taban ve sayı değerleri için logaritma değerleri uygun şekilde hesaplanarak her iki fonksiyon için hesaplanan değerler arasındaki farkın mutlak değeri (hata) son sütunda gösterilmiştir.

Tablo 1.-Tablo 4. değerlerinden görüleceği üzere $\mathrm{x}$ değeri arttıkça $\left|\log _{\mathrm{a}} \mathrm{x}-\operatorname{SLog}_{\mathrm{a}} \mathrm{x}\right|$ değerinin yani hatanın (göreceli olarak) arttığ görülmekle birlikte oluşan hatanın $\mathrm{x}=10^{30}$ gibi mühendislik uygulamaları açısından çok büyük sayı değeri için bile çok küu̧ük kaldığı görülmektedir (sirasılyla $5,10,50$ ve 500 taban değerleri için $\leq 5.34 \times 10^{-7}, \leq 5.18 \times 10^{-7}, \leq 2.70 \times 10^{-7}$ ve $\leq 1.78 \times 10^{-7}$ ) Dolayısıyla mevcut mühendislik ve matematik uygulamaları açısından oluşan hata değeri herhangi bir sorun teşkil etmemektedir. Şekil 4. ve Şekil 5. ile verilen grafikler de bu hususu pekiştirmektedir.

Benzer olarak Tablo 1.-Tablo 4. değerlerinden aynı sayı (x) değerleri için taban değerleri arttıkça oluşan $\left|\log _{\mathrm{a}} \mathrm{x}-\operatorname{SLog}_{\mathrm{a}} \mathrm{x}\right|$ hata değerinin de değiştiği görülmektedir. Ancak $\mathrm{x}=10^{30}$ gibi çok büyük sayı değeri dikkate alınacak olsa bile taban değeri arttıkça oluşan hatanın (her ne kadar hesaplamalarda taban ve sayı değerinden bağımsız olarak $10^{-7}$ mertebesinde gerçekleşiyor olsa bile) azaldığ 1 görülmektedir $\left(x=10^{30}\right.$ değeri için 5 ve 500 tabanları için sırasıyla $\leq 5.34 \times 10^{-7}$ ve $\left.\leq 1.78 \times 10^{-7}\right)$

- $\operatorname{SLog}_{\mathrm{a}} \mathrm{x}$ irrasyonel fonksiyonu elde edildikten sonra yine bilinen matematiksel tanım ve işlemler çerçevesinde bu fonksiyonun ters fonksiyonu oluşturulmak suretiyle $\operatorname{eSLog}_{\mathrm{a}} \mathrm{x}=\left[\mathrm{x} \cdot\left(\sqrt[3^{30}]{\mathrm{a}}-1\right)+1\right]^{2^{30}}$ üstel fonksiyonu elde edilmiştir.

Bu fonksiyon için de $\mathrm{y}=\mathrm{a}^{\mathrm{x}}$ üstel fonksiyonu ile birlikte analitik olarak grafiği oluşturulmuş ve Şekil 8. ile verilmiştir. Şekil 9. ile ise $\quad \mathrm{y}=\mathrm{a}^{\mathrm{x}}$ ve $\quad \operatorname{eSLog}_{\mathrm{a}} \mathrm{x}=[\mathrm{x} \cdot(\sqrt[20]{\mathrm{a}}-1)+1]^{2^{30}}$ fonksiyonunun grafikleri birlikte verilerek (16) ile elde edilen $\operatorname{eSLog}_{\mathrm{a}} \mathrm{x}=[\mathrm{x} \cdot(\sqrt[30]{\mathrm{a}}-1)+1]^{2^{30}}$ fonksiyonunun da $\mathrm{y}=\mathrm{a}^{\mathrm{x}}$ üstel fonksiyonun temsilinde kullanılabileceği gösterilmiştir. (Yer kaplamaması için üstel hesaplamalara ilişkin sayısal sonuçlar tablo olarak verilmemiştir.)

- Yukarıda belirtilen $\left|\log _{\mathrm{a}} \mathrm{x}-\operatorname{S} \log _{\mathrm{a}} \mathrm{x}\right|$ mutlak farkın (hatanın) $10^{-7}$ mertebesinde kalması sadece $\operatorname{SLog}_{\mathrm{a}} \mathrm{x}$ irrasyonel fonksiyonu için değil herhangi bir mertebeden türevi için de geçerlidir.

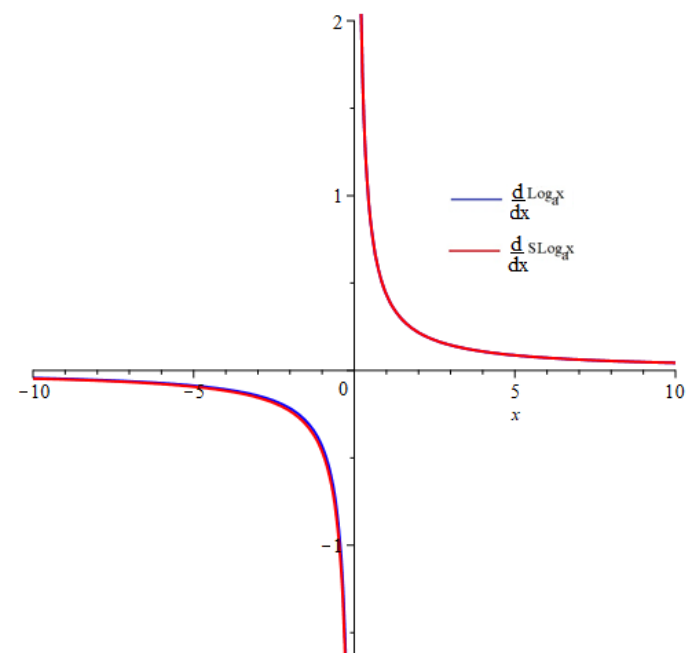

Şekil 10.- $a=10$ tabanı için $\frac{d\left(\log _{a} x\right)}{d x}$ ve $\frac{d\left(\operatorname{SLog}_{a} x\right)}{d x}$ grafikleri

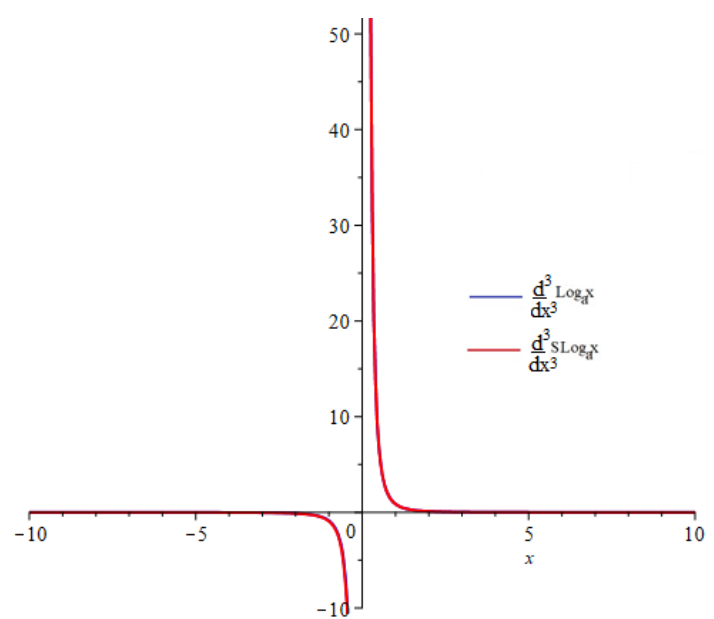

Şekil 11.- $a=10$ tabanı için $\frac{d^{3}\left(\log _{a} x\right)}{d x^{3}} v e \frac{d^{3}\left(\operatorname{SLog}_{a} x\right)}{d x^{3}}$ grafikleri

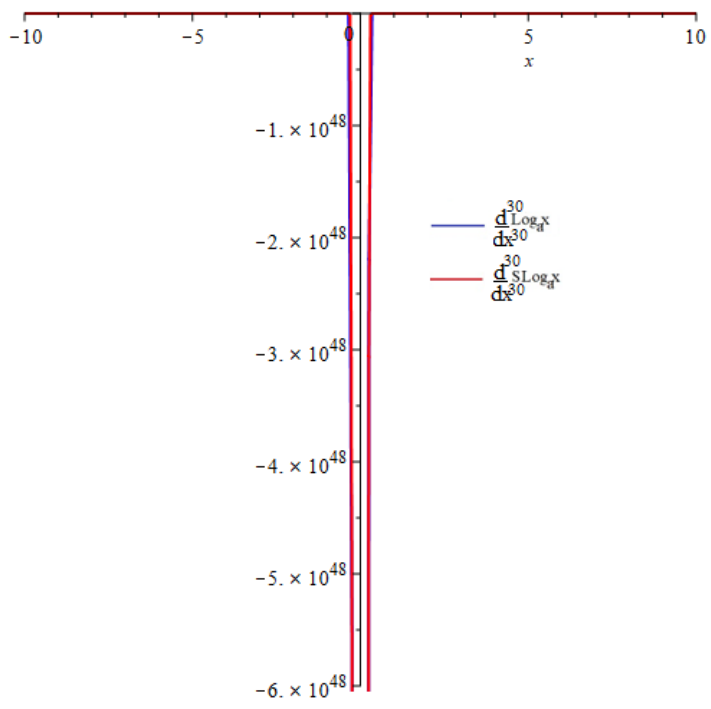

Şekil 12.- $a=10$ tabanı için $\frac{d^{30}\left(\log _{a} x\right)}{d x^{30}} v e \frac{d^{30}\left(\operatorname{SLog}_{a} x\right)}{d x^{30}}$ grafikleri 
Şekil 10.-Şekil 11. ile $a=10$ taban değeri için türev mertebesi sirasıyla $n=1,3$ ve 30 alınarak $\frac{d^{n}\left(\log _{a} x\right)}{d x^{n}}$ ve $\frac{d^{n}\left(\operatorname{SLog}_{a} x\right)}{d x^{n}}$ grafikleri bir arada verilmiştir.

- Bazı integral hesaplamalarının analitik olarak yapılması noktasında SLog $\operatorname{a}_{\mathrm{a}} \mathrm{x}$ fonksiyonunun kullanılması bazı kolaylıklar sağlayabilir. Örneğin:

$\mathrm{I}=\int(\operatorname{Ln} \mathrm{x})^{2} \mathrm{dx}$ belirsiz integralinin ilkelini analitik olarak bulabilmek için ardışık olarak iki kez kısmi integrasyon metodunu uygulamak gerekir.

$$
\begin{aligned}
& \mathrm{u}=(\operatorname{Ln} \mathrm{x})^{2} \quad ; \quad \mathrm{dv}=\mathrm{dx} \\
& \mathrm{du}=2 \cdot \operatorname{Ln} \mathrm{x} \cdot \frac{1}{\mathrm{x}} \cdot \mathrm{dx} \quad \mathrm{v}=\mathrm{x} \\
& \mathrm{I}=\mathrm{x} \cdot(\operatorname{Ln} \mathrm{x})^{2}-\int \mathrm{x} \cdot\left(2 \cdot \operatorname{Ln} \mathrm{x} \cdot \frac{1}{\mathrm{x}} \cdot \mathrm{dx}\right) \\
& \mathrm{I}=\mathrm{x} \cdot(\operatorname{Ln} \mathrm{x})^{2}-2 \int \operatorname{Ln} \mathrm{x} \cdot \mathrm{dx}
\end{aligned}
$$

olarak tekrar kısmi integrasyon metodunun uygulanması suretiyle;

$$
\begin{aligned}
& I=x \cdot(\operatorname{Ln} x)^{2}-2\left[x \cdot \operatorname{Ln} x-\int x \cdot\left(\frac{1}{x} d x\right)\right] \\
& I=x \cdot(\operatorname{Ln} x)^{2}-2\left[x \cdot \operatorname{Ln} x-\int d x\right] \\
& I=x \cdot(\operatorname{Ln} x)^{2}-2[x \cdot \operatorname{Ln} x-x+C] \\
& I=x \cdot(\operatorname{Ln} x)^{2}-2 x(\operatorname{Ln} x-1)+C_{1} \quad ; C_{1}=-2 C
\end{aligned}
$$

olarak bulunmuş olurdu. Eğer SLog $\mathrm{x}$ fonksiyonu ile integrasyon işlemi yapılmış olsaydı;

$I=\int\left(\frac{x^{n}-1}{a^{n}-1}\right)^{2} \cdot d x=\frac{1}{\left(a^{n}-1\right)^{2}} \int\left(x^{n}-1\right)^{2} \cdot d x \quad ; n=\frac{1}{2^{30}}$

$I=\frac{1}{\left(a^{n}-1\right)^{2}} \int\left(x^{2 n}-2 \cdot x^{n}+1\right) \cdot d x$

$I=\frac{1}{\left(a^{n}-1\right)^{2}}\left(\frac{x^{2 n+1}}{2 n+1}-2 \cdot \frac{x^{n+1}}{n+1}+x\right)+C_{2} \quad ; n=\frac{1}{2^{30}}$

şeklinde elde edilmiş olurdu. Yukarıda verilen örnek uygulamadan da görüleceği üzere bu tür integrallerin analitik çözümlerine ilişkin olarak $\operatorname{SLog}_{\mathrm{a}} \mathrm{x}$ fonksiyonunu kullanmak gerek zaman gerekse işlem açısından avantaj sağlamaktadır. $\mathrm{Bu}$ avantaj $\int \mathrm{x}^{\mathrm{p}} \cdot(\operatorname{Ln} \mathrm{x})^{\mathrm{q}} \cdot \mathrm{dx} \quad\left(\mathrm{p}, \mathrm{q} \in \mathrm{Z}^{+}\right)$tarzinda integrallerin çözümünde daha da artmaktadır.
- Değişik taban ve sayı değerleri için $\log _{a} x$ ve $\operatorname{S} \log _{a} x$

\begin{tabular}{|c|c|c|c|}
\hline$x$ & $\log _{10} x$ & $\operatorname{SLog}_{10} x$ & $\left|\log _{10} x-\operatorname{SLog}_{10} x\right|$ \\
\hline 1 & 0,000000000 & 0,000000000 & 0,000000000 \\
\hline 5 & 0,698970004 & 0,698970008 & 0,000000004 \\
\hline 10 & 1,000000000 & 1,000000000 & 0,000000000 \\
\hline 50 & 1,698970004 & 1,698970008 & 0,000000004 \\
\hline 100 & 2,000000000 & 2,000000000 & 0,000000000 \\
\hline 500 & 2,698970004 & 2,698970008 & 0,000000004 \\
\hline 1.000 & 3,000000000 & 3,000000000 & 0,000000000 \\
\hline 5.000 & 3,698970004 & 3,698970008 & 0,000000004 \\
\hline 10.000 & 4,000000000 & 4,000000000 & 0,000000000 \\
\hline 50.000 & 4,698970004 & 4,698970008 & 0,000000004 \\
\hline 100.000 & 5,000000000 & 5,000000000 & 0,000000000 \\
\hline 500.000 & 5,698970004 & 5,698970008 & 0,000000004 \\
\hline 1.000 .000 & 6,000000000 & 6,000000000 & 0,000000000 \\
\hline 5.000 .000 & 6,698970004 & 6,698970008 & 0,000000004 \\
\hline 10.000 .000 & 7,000000000 & 7,000000000 & 0,000000000 \\
\hline 50.000 .000 & 7,698970004 & 7,698970008 & 0,000000004 \\
\hline 100.000 .000 & 8,000000000 & 8,000000000 & 0,000000000 \\
\hline 500.000 .000 & 8,698970004 & 8,698970008 & 0,000000004 \\
\hline 1.000 .000 .000 & 9,000000000 & 9,000000000 & 0,000000000 \\
\hline 5.000 .000 .000 & 9,698970004 & 9,698970008 & 0,000000004 \\
\hline 10.000 .000 .000 & 10,000000000 & 10,000000000 & 0,000000000 \\
\hline 50.000 .000 .000 & 10,698970004 & 10,698970008 & 0,000000004 \\
\hline 100.000 .000 .000 & 11,000000000 & 11,000000000 & 0,000000000 \\
\hline . & . & . & . \\
\hline . & . & . & . \\
\hline . & . & & \\
\hline $10^{\wedge} 30$ & 30,000000000 & 30,000000518 & 0,000000518 \\
\hline
\end{tabular}
fonksiyonları için hesaplanan değerler ve $\left|\log _{a} x-S \log _{a} x\right|$ değeri (hata) Tablo 1.-Tablo 4. ile verilmiştir.

Tablo 1. $a=5$ tabanı için hesaplanan $\log _{a} x$ ve $\operatorname{SLog}_{a} x$ değerleri ve mutlak fark (hata)

\begin{tabular}{|c|c|c|c|}
\hline $\mathbf{x}$ & $\log _{5} \mathrm{x}$ & $\mathrm{SLog}_{5} \mathrm{x}$ & $\log _{5} \mathrm{x}-\operatorname{SLog}_{5} \mathrm{x} \mid$ \\
\hline 1 & 0,000000000 & 0,000000000 & 0,000000000 \\
\hline 5 & 1,000000000 & 1,000000000 & 0,000000000 \\
\hline 10 & 1,430676558 & 1,430676551 & 0,000000007 \\
\hline 50 & 2,430676558 & 2,430676551 & 0,000000007 \\
\hline 100 & 2,861353116 & 2,861353102 & 0,000000014 \\
\hline 500 & 3,861353116 & 3,861353102 & 0,000000014 \\
\hline 1.000 & 4,292029674 & 4,292029653 & 0,000000021 \\
\hline 5.000 & 5,292029674 & 5,292029653 & 0,000000021 \\
\hline 10.000 & 5,722706232 & 5,722706204 & 0,000000028 \\
\hline 50.000 & 6,722706232 & 6,722706204 & 0,000000028 \\
\hline 100.000 & 7,153382790 & 7,153382756 & 0,000000034 \\
\hline 500.000 & 8,153382790 & 8,153382756 & 0,000000034 \\
\hline 1.000 .000 & 8,584059348 & 8,584059307 & 0,000000041 \\
\hline 5.000 .000 & 9,584059348 & 9,584059307 & 0,000000041 \\
\hline 10.000 .000 & 10,014735906 & 10,014735858 & 0,000000048 \\
\hline 50.000 .000 & 11,014735906 & 11,014735858 & 0,000000048 \\
\hline 100.000 .000 & 11,445412464 & 11,445412409 & 0,000000055 \\
\hline 500.000 .000 & 12,445412464 & 12,445412409 & 0,000000055 \\
\hline 1.000 .000 .000 & 12,876089022 & 12,876088960 & 0,000000062 \\
\hline 5.000 .000 .000 & 13,876089022 & 13,876088960 & 0,000000062 \\
\hline 10.000 .000 .000 & 14,306765580 & 14,306765512 & 0,000000068 \\
\hline 50.000 .000 .000 & 15,306765580 & 15,306765512 & 0,000000068 \\
\hline 100.000 .000 .000 & 15,737442138 & 15,737442063 & 0,000000075 \\
\hline$\cdot$ &. &. &. \\
\hline$\cdot$ &. &. &. \\
\hline$\cdot$ &. & &. \\
\hline $10^{\wedge} 30$ & 42,92029674 & 42,920297276 & 0,000000534 \\
\hline
\end{tabular}

Tablo 2. $a=10$ tabanı için hesaplanan $\log _{a} x$ ve $S \log _{a} x$ değerleri ve mutlak fark (hata) 
Tablo 3. $a=50$ tabanı için hesaplanan $\log _{a} \mathrm{x}$ ve $\operatorname{SLog}_{\mathrm{a}} \mathrm{x}$ değerleri ve mutlak fark (hata)

\begin{tabular}{|c|c|c|c|}
\hline$x$ & $\log _{50} x$ & $\operatorname{SLog}_{50} \mathrm{x}$ & $\left|\log _{50} \mathrm{x}-\mathrm{SLog}_{50} \mathrm{x}\right|$ \\
\hline 1 & 0,000000000 & 0,000000000 & 0,000000000 \\
\hline 5 & 0,411408090 & 0,411408091 & 0,000000001 \\
\hline 10 & 0,588591910 & 0,588591909 & 0,000000001 \\
\hline 50 & 1,000000000 & 1,000000000 & 0,000000000 \\
\hline 100 & 1,177183820 & 1,177183818 & 0,000000002 \\
\hline 500 & 1,588591910 & 1,588591909 & 0,000000001 \\
\hline 1.000 & 1,765775730 & 1,765775727 & 0,000000003 \\
\hline 5.000 & 2,177183820 & 2,177183810 & 0,000000010 \\
\hline 10.000 & 2,354367640 & 2,354367636 & 0,000000004 \\
\hline 50.000 & 2,765775730 & 2,765775727 & 0,000000003 \\
\hline 100.000 & 2,942959550 & 2,942959545 & 0,000000005 \\
\hline 500.000 & 3,354367640 & 3,354367636 & 0,000000004 \\
\hline 1.000 .000 & 3,531551460 & 3,531551453 & 0,000000007 \\
\hline 5.000 .000 & 3,942959550 & 3,942959545 & 0,000000005 \\
\hline 10.000 .000 & 4,120143370 & 4,120143362 & 0,000000008 \\
\hline 50.000 .000 & 4,531551460 & 4,531551453 & 0,000000007 \\
\hline 100.000 .000 & 4,708735281 & 4,708735271 & 0,000000010 \\
\hline 500.000 .000 & 5,120143370 & 5,120143362 & 0,000000008 \\
\hline 1.000 .000 .000 & 5,297327191 & 5,297327180 & 0,000000011 \\
\hline 5.000 .000 .000 & 5,708735281 & 5,708735271 & 0,000000010 \\
\hline 10.000 .000 .000 & 5,885919101 & 5,885919089 & 0,000000012 \\
\hline 50.000 .000 .000 & 6,297327191 & 6,297327180 & 0,000000011 \\
\hline 100.000 .000 .000 & 6,474511011 & 6,474510998 & 0,000000013 \\
\hline . & . & . & . \\
\hline . & . & . & . \\
\hline . & . & . & . \\
\hline $10^{\wedge} 30$ & 17,6577573 & 17,657757572 & 0,000000270 \\
\hline
\end{tabular}

Tablo 4. $\mathrm{a}=500$ tabanı için hesaplanan $\log _{\mathrm{a}} \mathrm{x}$ ve $\operatorname{SLog}_{\mathrm{a}} \mathrm{x}$ değerleri ve mutlak fark (hata)

\begin{tabular}{|c|c|c|c|}
\hline$x$ & $\log _{500} x$ & $\operatorname{SLog}_{500} \mathrm{x}$ & $\left|\log _{500} x-\operatorname{SLog}_{500} x\right|$ \\
\hline 1 & 0,000000000 & 0,000000000 & 0,000000000 \\
\hline 5 & 0,258976574 & 0,258976575 & 0,000000001 \\
\hline 10 & 0,370511713 & 0,370511713 & 0,000000000 \\
\hline 50 & 0,629488287 & 0,629488287 & 0,000000000 \\
\hline 100 & 0,741023426 & 0,741023425 & 0,000000001 \\
\hline 500 & 1,000000000 & 1,000000000 & 0,000000000 \\
\hline 1.000 & 1,111535139 & 1,111535138 & 0,000000001 \\
\hline 5.000 & 1,370511713 & 1,370511713 & 0,000000000 \\
\hline 10.000 & 1,482046853 & 1,482046851 & 0,000000002 \\
\hline 50.000 & 1,741023426 & 1,741023425 & 0,000000001 \\
\hline 100.000 & 1,852558566 & 1,852558563 & 0,000000003 \\
\hline 500.000 & 2,111535139 & 2,111535138 & 0,000000001 \\
\hline 1.000 .000 & 2,223070279 & 2,223070276 & 0,000000003 \\
\hline 5.000 .000 & 2,482046853 & 2,482046851 & 0,000000002 \\
\hline 10.000 .000 & 2,593581992 & 2,593581989 & 0,000000003 \\
\hline 50.000 .000 & 2,852558566 & 2,852558563 & 0,000000003 \\
\hline 100.000 .000 & 2,964093705 & 2,964093701 & 0,000000004 \\
\hline 500.000 .000 & 3,223070279 & 3,223070276 & 0,000000003 \\
\hline 1.000 .000 .000 & 3,334605418 & 3,334605414 & 0,000000004 \\
\hline 5.000 .000 .000 & 3,593581992 & 3,593581989 & 0,000000003 \\
\hline 10.000 .000 .000 & 3,705117131 & 3,705117127 & 0,000000004 \\
\hline 50.000 .000 .000 & 3,964093705 & 3,964093701 & 0,000000004 \\
\hline 100.000 .000 .000 & 4,075628844 & 4,075628839 & 0,000000005 \\
\hline . & . & . & . \\
\hline . & . & . & . \\
\hline . & . & . &. \\
\hline $10^{\wedge} 30$ & 11,11535139 & 11,115351572 & 0,000000178 \\
\hline
\end{tabular}

amaçlanarak $\operatorname{SLog}_{\mathrm{a}} \mathrm{x}$ irrasyonel fonksiyonu önerilmiş ve elde edilmiştir. Şekil 4., Şekil 5. grafikleri ve Tablo 1.-Tablo 4. sayısal sonuçlarından $\operatorname{SLog}_{\mathrm{a}} \mathrm{x}$ irrasyonel fonksiyonunun matematik, mühendislik ve diğer bilim dallarında yaygın kullanımı olan $\log _{\mathrm{a}} \mathrm{x}$ fonksiyonu ile aynı işleve sahip olduğu gösterilmiş, her iki fonksiyon için de bulunan değerler arasında oluşan mutlak farkın (hatanın) seçilen taban (a) ve sayı değerlerinden (x) bağımsız olmak üzere

$$
\left|\log _{\mathrm{a}} \mathrm{x}-\mathrm{S} \log _{\mathrm{a}} \mathrm{x}\right|<10^{-7}
$$

olarak gerçekleştiği, SLog $\log _{\mathrm{a}} \mathrm{x}$ irrasyonel fonksiyonunun $\log _{\mathrm{a}} \mathrm{x}$ fonksiyonunun temsilinde kullanilabileceği, $\log _{\mathrm{a}} \mathrm{x}$ fonksiyonunun kendisi (veya üslü ifadeleri) dikkate alındığında bazı durumlarda analitik olarak integral alma işlemlerini $\operatorname{SLog}_{\mathrm{a}} \mathrm{x}$ ile yapmanın avantaj sağlayacağı gösterilmiştir.

- $\mathrm{Bu}$ çalışmada önerilmiş ve elde edilmiş olan $\operatorname{SLog}_{\mathrm{a}} \mathrm{x}$ ve eSLog $\operatorname{ar}_{\mathrm{a}} \mathrm{x}$ fonksiyonları ile $\log _{\mathrm{a}} \mathrm{x}$ ve $\mathrm{y}=\mathrm{a}^{\mathrm{x}}$ fonksiyonları için hesaplanan değerler arasındaki fark (hata), mühendislik ve matematik uygulamaları açısından sorun teşkil etmeyecek derecede çok küçük $\left(<10^{-7}\right)$ kalmakta, gerçek değerler ile çelişmemektedir.

- Bu çalışmanın amacı, temelde $\log _{\mathrm{a}} \mathrm{x}$ fonksiyonunun irrasyonel $\operatorname{SLog}_{\mathrm{a}} \mathrm{x}$ fonksiyonu ile temsil edilmesine yönelik olduğundan başlangıç olarak $\log _{\mathrm{a}} \mathrm{x}$ fonksiyonu ile yapılan logaritma hesaplarının, grafiklerin elde edilmesi ve özellikle $\log _{\mathrm{a}} \mathrm{x}$ ihtiva eden, analitik olarak çözülmesi zaman alan ve çok işlem gerektiren integrallerin alınmasının daha kolay bir şekilde yapılabilmesi hedeflenmiş ve $\operatorname{SLog}_{\mathrm{a}} \mathrm{x}$ fonksiyonu ile bazı integrallerin analitik olarak daha kolay çözülebileceği gösterilmiştir.

- Ancak bu çalışmada esas olarak $\log _{a} \mathrm{x}$ ve $\mathrm{y}=\mathrm{a}^{\mathrm{x}}$ fonksiyonlarının temsiline ilişkin olarak $\operatorname{SLog}_{a} x$ ve $\operatorname{eSLog}_{a} x$ fonksiyonlarının elde edilmesi hedeflendiğinden $\operatorname{SLog}_{\mathrm{a}} \mathrm{x}$ ve $\mathrm{eSLog}_{\mathrm{a}} \mathrm{x}$ fonksiyonlarının analitik olarak incelenmeleri, seri açılımları ve bu doğrultuda $\log _{\mathrm{a}} \mathrm{x}$ fonksiyonu ile olan yakınsaklık durumlarının irdelenmesi, $\log _{\mathrm{a}} \mathrm{x}$ ve $\mathrm{y}=\mathrm{a}^{\mathrm{x}}$ fonksiyonlarını içeren adi veya kısmi diferansiyel denklemlere benzer olarak aynı çalışmaların $\operatorname{SLog}_{\mathrm{a}} \mathrm{x}$ ve $\operatorname{eSLog}_{\mathrm{a}} \mathrm{x}$ fonksiyonları için de yapılması, gerekli çıkarımların, olası sınırlılıkların belirlenmesi, kompleks sayıların logaritmasının hesaplanması bu çalışmada yapılmayan fakat bu çalışmamının devamı olarak yapılması düşünülen başlıklar olarak sayılabilir.

- Bütün bu hususlar dahilinde $\operatorname{SLog}_{a} \mathrm{x}$ ve eSLog $_{\mathrm{a}} \mathrm{x}$ ile önerilen fonksiyonların bu çalışma ile ilk kez önerilmiş ve elde edilmiş olmaları, matematiğin en temel tanım, kavram ve fonksiyonları için bile süreç içerisinde yeni öneri, yöntem ve yorumların yapılabileceği fikrini desteklemesi açısından önemlidir.

\section{Sonuç}

- $\mathrm{Bu}$ çalışmada logaritma hesabı için kullanılan $\log _{\mathrm{a}} \mathrm{x}$ fonksiyonunun irrasyonel bir fonksiyon ile temsil edilebileceği 


\section{Kaynakça}

Aleksandrov, A. G., (2014). Residues of Logarithmic Differential Forms in Complex Analysis and Geometry, Analysis in Theory and Applications, Vol. 30, No. 1, 34-50.

Andrews G. E., Askey R., Roy R. (1999). Special Functions, Encyclopedia of Mathematics and its Applications, 71, Cambridge University Press

Boyer, C. B. and Merzbach, U. C. (1991). Invention of Logarithms., A History of Mathematics, 2nd ed. New York: Wiley, 312-313.

Bruce, I., (2000), Napier's logarithms., American Journal of Physics, 68(2):148-154

Fauvel, J., (2000). John Napier 1550-1617. EMS Newsletter, 38:24-25, (Reprinted pp. 1, 6-8 of CMS Notes-Notes de la $S M C$, volume 33, issue 6, October 2001)

Gautschi, W. (2008). On Euler's attempt to compute logarithms by interpolation: A commentary to his letter of February 16, 1734 to Daniel Bernoulli, Journal of Computational and Appllied Mathematics, 219, no.2, 408-415.

Havil, J. (2003). The Baron's Wonderful Canon., 1.2 in Gamma: Exploring Euler's Constant. Princeton, NJ: Princeton University Press, 4-11.

Kathleen, M., Montelle, C., (2015). Logarithms: The Early History of a Familiar Function - John Napier Introduces Logarithms, Mathematical Association of America.

Kreyszig E. (1993). Advanced Engineering Mathematics, John Wiley \& Sons, 7th Edition.

Matala-Aho T., Vaananen Keijo, Zudilin Wadim. (2005). New Irrationality Measures for q-Logarithms, Mathematics of Computation, v. 75, Number 254, 879-889.

Mathews J. H. (1992). Numerical Methods for Mathematics, Science and Engineering, Prentice-Hall Inc., 2nd Ed.

Koelink, E., Assche, W. V. (2009). Leonhard Euler and a qanalogue of the logarithm. , American Mathematical Society, v.137, n.5, 1663-1676.

Nofal, C. P. (2006) Proof that the Natural Logarithm Can Be Represented by the Gaussian Hypergeometric Function.

Pol D., (2018), On the values of logarithmic residues along curves, Ann. Inst. Fourier Grenoble, 68, 2, 725-766.

Rice B., Gonzales-Velasco E., Corrigan A. (2017). John Napier. In The Life and Works of John Napier, Springer.

Tajima. S., Nabeshima, K., (2021). Computing Regular Meromorphic Differential Forms via Saito's Logarithmic Residues, Symmetry, Integrability and Geometry: Methods and Applications, Sigma 17.

Thomas, G. B., Maurice D. W., Joel H. R. (2010). Thomas' Calculus, 12th Edition, Pearson. 